\title{
Implementación del aprendizaje cooperativo como estrategia didáctica para la construcción del conocimiento, en el área de la logística, en estudiantes de la carrera de ingeniería industrial
}

\author{
Implementation of cooperative learning as a didactic strategy aiming at knowledge \\ building in the logistics area, for Industrial Engineering students
}

\author{
Jose Roig-Zamora ${ }^{1}$ \\ Escuela de Ingeniería Industrial, \\ Facultad de Ingeniería, \\ Universidad de Costa Rica \\ San José, Costa Rica \\ jose.roig@ucr.ac.cr
}

\begin{abstract}
Recibido: 30 octubre $2012 \quad$ Aceptado: 13 marzo $2014 \quad$ Corregido: 25 marzo 2014
\end{abstract}
Resumen: Este artículo desarrolla una estrategia didáctica que se implementó para los estudiantes de la carrera de Ingeniería Industrial que se enfrentan por primera vez a temáticas de logística. En este caso, el curso de Logística I, le ofrece al estudiantado la fundamentación teórica sobre esta temática y asimismo brinda espacios sobre su aplicación mediante un trabajo de campo. Con el propósito de innovar los procesos de construcción del conocimiento respecto a las temáticas que se suscitan en el curso, se propone la implementación de una estrategia didáctica basada en el Aprendizaje Cooperativo, para diseñar una situación de aprendizaje que le permita al estudiantado potenciar la aplicación del conocimiento a entornos complejos, reales y concretos. Por lo tanto, considerando los elementos teóricos en relación con la logística, los estudiantes de manera grupal deben desarrollar una propuesta ficticia y detallada de un negocio, para un potencial inversionista que pretende abrir una cadena de restaurantes en Costa Rica. Para esta intención, se propone el Modelo del Aprendizaje Cooperativo con el objetivo de generar y fomentar la construcción de conocimiento y la aplicación de la teoría logística en la práctica, esto mediante el desarrollo cooperativo del alumnado hacia el armado de su modelo de negocio y el dominio de sus diversas variables logísticas. Mediante la aplicación de esta estrategia en la Ingeniería Industrial se demuestra que el aprendizaje cooperativo potencia la capacidad del alumnado universitario para la correcta asimilación de las temáticas teóricas y la posibilidad de aplicación de estas a un contexto real y complejo.

Palabras claves: estrategia didáctica, colaboración, Aprendizaje Cooperativo, logística, modelo de negocio, conocimiento aplicado.

Abstract: This article presents a didactic strategy which was developed with Industrial Engineering students who encounter basic concepts on logistics for the first time. The Logistics I course offers the appropriate theoretical framework needed to apply the concepts through a fieldwork project. With the purpose of innovating and improving the learning processes within the course, the implementation of a didactic strategy based on the Cooperative Learning Approach is proposed to design a learning experience which would allow the students to foster and implement knowledge to complex situations

1 Máster en Logística de la Universidad Nacional de Cuyo, Argentina. Master en Ciencias, Tecnología y Organización (Diseño Global, mención Innovación y Diseño de Sistemas Industriales) de la Universidad de Lorraine, Francia. Profesor de la Escuela de Ingeniería Industrial, Facultad de Ingeniería, Universidad de Costa Rica. 
and contexts. Considering the theoretical elements related to the logistics field, the students would have to develop a detailed business model proposal for a potential investor that intended to open a chain of restaurants in Costa Rica. The objective of Cooperative Learning Approach as a proposal is to generate their knowledge construction process by applying the logistic theory in real life situations, using the student's cooperative development to construct the business model proposal, therefore, reaching a better management of its diverse logistics variables. By means of this innovation, applied to the Industrial Engineering field, it was possible to demonstrate that the Cooperative Learning strategies reinforced university students' capacity to assimilate theoretical subjects and then to apply them into plausible complex contexts.

Keywords: Didactic strategy, collaboration, Cooperative Learning, logistics, business model-applied knowledge.

\section{Introducción}

Como docentes universitarios, es constante la preocupación sobre la manera en que los estudiantes procesan el conocimiento y cómo estos lo relacionan con la práctica. Por ello, dentro de la reflexión que hacemos los docentes sobre nuestro rol como mediadores en el proceso de aprendizaje nos cuestionamos lo siguiente:

¿Cómo logramos que el estudiantado logre una mejor comprensión de la teoría y aplicación de la misma una situación real?

¿Cuáles serían las estrategias didácticas que permiten relacionar la teoría y la práctica a nivel universitario?

Específicamente, en la carrera de Ingeniería Industrial de la Universidad de Costa Rica, el curso de Logistica de la Cadena de Valor desarrolla la fundamentación teórica y básica sobre temas de logística. En dicho curso se pretende que el estudiante conozca el estado del arte concerniente a la logística y logre aplicarla en cualquier entorno industrial.

De acuerdo con la experiencia obtenida como docente en el curso, he visualizado la dificultad de los educandos para relacionar los conceptos teóricos en la práctica y es notable la poca reflexión y análisis que se realiza sobre la aplicación y contextualización real del conocimiento en el campo de trabajo, esto a veces por la complejidad del acceso del estudiante a entornos de aplicación real, principalmente por la confidencialidad de los datos que varias empresas exigen y que marcan una barrera para el estudiantado.

Por lo tanto, con el propósito de favorecer los procesos de construcción del conocimiento del alumnado, se implementó una estrategia didáctica innovadora basada en el aprendizaje cooperativo hacia el entendimiento de un negocio en concreto como base para entender la teoría aplicada en un contexto industrial.

Específicamente, el objetivo es implementar una estrategia didáctica innovadora en la Ingeniería Industrial, mediante el uso del aprendizaje cooperativo como herramienta para lograr la construcción de conocimiento respecto a los temas de logística, en estudiantes con contacto primerizo hacia el tema.

El curso de Logística de la Cadena de Valor I, es el primer contacto de los estudiantes en la carrera de Ingeniería Industrial con la temática de logística. Esta población estudiantil posee pocos conocimientos 
sobre el tema y asimismo muestran un leve desconocimiento del medio industrial. Además, es importante señalar que los contenidos que se operacionalizan en el curso permiten a su vez una gran factibilidad de aplicación en la práctica, es decir, deben ser puestos en práctica para que se logren entender.

Sin embargo, esta aplicación teórica debe ser mediada con estrategias didácticas que permitan la relación teórico-práctica dentro de un entorno funcional y atractivo para el estudiantado.

\section{Referente teórico}

La estrategia docente implementada se centra específicamente en el aprendizaje cooperativo, el cual es un modelo pedagógico basado en el trabajo en grupo para la construcción del conocimiento. Este modelo está fundamentado en el enfoque pedagógico socio histórico de Vigotsky, el cual destaca la importancia de las interacciones sociales, el lenguaje, la cultura, en el proceso de enseñanza-aprendizaje (Smagorinsky, 2007).

Algunos autores lo llaman de manera indistinta como trabajo colaborativo o aprendizaje cooperativo (Ferreiro, Calderón, 2007).

Según Ferreiro y Calderón (2007), este aprendizaje tiene tres aspectos medulares, mismos que se conocen como el $\mathrm{ABC}$ del aprendizaje cooperativo:

- A (Actividad)

- B (Bidireccionalidad)

- C (Colaboración)

La A, hace referencia a la actividad, al subrayar la necesidad de hacer participar a los alumnos en su proceso de enseñanza-aprendizaje. Es la actividad que aprende, tanto a nivel interno como externo, es decir, está relacionado con los procesos internos psicológicos que se pueden evidenciar en la actividad externa de la persona.

En el aprendizaje cooperativo tanto los momentos de trabajo individual y por otra parte la interacción en el trabajo con otros (que es la interactividad necesaria para aprender), son esenciales, de ahí la importancia de alternarlos, potenciando el trabajo individual y el de equipo y los respectivos resultados. Lo anterior, se puede reducir en que el buen trabajo con otros requiere de un esfuerzo individual. En este aspecto se hace hincapié en que si bien el aprendizaje cooperativo se ha utilizado en muchas aplicaciones docentes, este tipo de actividad que se plantea en este artículo es la primera vez que lo experimento en el ámbito de la Escuela de Ingeniería Industrial (tanto como profesor como estudiante en su momento), pues el diseño de la actividad al ponerles el mismo objetivo a todos los grupos de estudiantes y al realizar un taller cooperativo-comparativo, permite la colaboración entre ellos y por ende hace posible profundizar más el conocimiento del modelo de negocio, ya que todos apuntan a un único fin (dando base a la necesidad de colaboración).

La B se relaciona con la bi-direccionalidad necesaria en el proceso de enseñanza aprendizaje, entre el que guía y orienta la actividad y el aprendiz. Este modelo plantea 
que la manera de guiar es a través de la mediación. El rol del profesor es de mediador más que un trasmisor de conocimiento, esto debido a que se entiende como más productivo que el estudiante llegue a encontrar las respuestas más que ver a su profesor como la fuente de solución. Según Ferreiro y Calderón (2007) el profesor mediador es la persona que al relacionarse con otra u otras es capaz de:

- Favorecer el aprendizaje.

- Estimular el desarrollo de potencialidades

- Corregir funciones cognitivas deficientes

En este sentido, el proceso de mediación parte de la premisa de que es posible la modificabilidad cognitiva así como también la afectiva en la persona, la cual se propicia a través de la interrelación entre los sujetos. Este proceso se caracteriza por ser intencionado y de reciprocidad entre los miembros de un equipo, además de compartir experiencias significativas que trascienden el aquí y el ahora. Para el buen éxito de las tareas, en el aprendizaje cooperativo el profesor tiene un rol importante de seguimiento constante, comunicación clara de las tareas, guía para enfrentar dificultades y promoción de un sistema de evaluación (Li, 2001). En este aspecto la metodología planteada permite que el profesor sea visto como mediador pues es el estudiante el que se vuelve experto en el entorno del negocio y en el cómo aplicar la teoría a dicha realidad; es mediante esta aplicación que se acelera el proceso de asimilación del concepto logístico, pues el estudiante debe interpretarlo, dominarlo y acomodarlo para así lograr una correcta aplicación del concepto a la realidad del negocio que estudia.

Por otro lado, la $\mathrm{C}$ en el aprendizaje hace alusión a la colaboración entre las personas para aprender en clase. Se pueden identificar diferentes formas de relación entre los estudiantes para aprender. En este sentido, está el individualista, es decir, cada uno en lo suyo sin importar lo del otro, por lo tanto, no es trascendental que los estudiantes se comuniquen y el profesor utiliza método expositivo para enseñar. Otra forma es la competitiva, en la que cada uno de los miembros de un grupo de estudiantes percibe que puede obtener el objetivo de enseñanza si y sólo si, el resto de los estudiantes no lo obtienen.

Por último, el tercer tipo de relación para aprender es la colaboración, que se da cuando cada uno de los que integran el equipo percibe que pueden lograr el objetivo si, y solo si, todos trabajan juntos y cada quien aporta su parte. La colaboración entre los miembros de un grupo de estudiantes es la piedra angular de esta forma de organización del proceso aprendizaje. A lo largo de la propuesta, esta particularidad se logra al ser un proyecto real, pues al ser real requiere de análisis detallado y por ende se requiere repartir el trabajo para salir con él de manera cooperativa.

El aprendizaje cooperativo, también denominado trabajo en grupos cooperativos, es una forma distintiva de trabajo grupal que se caracteriza por que los grupos abordan la resolución de una misma tarea o problema trabajando en conjunto. La tarea es abordada por estudiantes que se encuentran en un mismo nivel de conocimiento o discretamente diferente, gracias a la acción conjunta, los resultados logrados son distintos a los que se lograrían si cada integrante trabajara individualmente. Debido a esto es que el aporte de cada miembro es muy valioso. 
Este aprendizaje brinda a los estudiantes una atmósfera de comunicación donde pueden expresarse libremente y favorece los procesos de tutorías y ayuda al interior del grupo. "Cada miembro tiene responsabilidades definidas y es corresponsable del aprendizaje de los otros, pues los grupos evalúan constantemente su desempeño y la calidad de su trabajo para la búsqueda de la mejora continua." (Amaya, s.f., p.4) El aprendizaje permite a los estudiantes el desarrollo de competencias relacionadas con la comunicación y habilidades sociales (integración, respeto, tolerancia, solidaridad) mismos que son fundamentales para interacción con otros (Baumberger-Henry, 2005).

Por lo que al trabajar de manera cooperativa se crean mayores relaciones positivas entre los estudiantes que en forma competitiva o individual. Los miembros se influencian unos con otros y logran un más alto nivel de desempeño en el trabajo cooperativo que en el trabajo individual.

Según Tenutto y Klinoff (2005) uno de los aspectos primordiales del aprendizaje cooperativo deriva de la interdependencia positiva, pues cada miembro del grupo tiene claro que los esfuerzos que hace no solo beneficiarán a los restantes integrantes sino que a él mismo. Esta interdependencia logra fomentar en el grupo la conveniencia de que todos los integrantes tengan clara la teoría para que logren aportar al grupo, lo que genera que todos esté al día. La interdependencia positiva, Tenutto y Klinoff (2005) las tipifica y ejemplifica en cinco vertientes: la interdependencia de metas, la interdependencia de tareas, interdependencia de recursos, interdependencia de roles y la interdependencia de premios.

Para sacar una tarea en común hay varios elementos que deben aparecer en un grupo cooperativo. Estos elementos son descritos por Tenutto y y Klinoff (2005) como habilidades cooperativas. Específicamente se habla de comunicación e interacción con otros, escucha activa, hablar por turnos, compartir e intercambiar ideas, sintetizar ideas, opinar y expresar el pensamiento y dar apoyo y/o aceptación hacia las ideas necesarias de considerar para llegar al resultado buscado.

En el trabajo de grupo siempre existe un gran riesgo de que unos estudiantes se aprovechen del trabajo de otros. Es decir, que unos estudiantes que usualmente ponen un menor esfuerzo se vean beneficiados por el esfuerzo extra de otros colegas. Uno de los principios en el cual debe insistir el profesor es el que menciona Tenutto y Klinoff. (2005), respecto al principio de responsabilidad individual el cual consiste en que cada individuo debe dominar y ejecutar la parte del trabajo por la que se hizo responsable dentro del grupo de aprendizaje cooperativo. Es decir, la responsabilidad es individual como grupal si se quiere agresivamente alcanzar el objetivo. En este aspecto, al delegar en el grupo de trabajo una tarea con carga de trabajo sustancial, origina en el grupo una presión de sacar la tarea en conjunto, y es esta misma presión la que lleva al grupo a presionar implícita y explícitamente a cada miembro a hacerse cargo de lo que se le asignó (una especia de presión colectiva cooperativa es la que mueve a los grupos, más que la presencia del profesor "vigilante").

De acuerdo a todo lo anterior, se podrían establecer las siguientes pautas de una metodología según el aprendizaje cooperativo: 
- El profesor plantea de manera clara y concisa lo que espera que sus estudiantes aprendan, discutan, construyan y propongan desde el punto de vista conceptual, asimismo, cuales habilidades sociales está buscando desarrollar de manera particular con la experiencia de aprendizaje.

- Se establece un esquema de trabajo, en el cual se hace una estructuración de la clase en grupos heterogéneos, así como las pautas para trabajar los contenidos con la ayuda de sus iguales y con la orientación del profesor si es necesario.

- Se debe establecer objetivos claros de trabajo en el desarrollo de la clase. Es importante considerar que el eje fundamental de los espacios de trabajo es la ayuda entre los miembros del grupo. Asimismo, se solicita ayuda al profesor en caso de que el grupo no pueda resolverlo por sí mismo, esto con el fin de promover la autonomía.

- En relación con el rol del profesor, este no interviene de manera magistral, sólo para facilitar el proceso de aprendizaje y fungir como guía en la construcción del conocimiento. En este caso, se promueve que sus explicaciones se realicen a partir de las preguntas o dudas que los estudiantes planteen.

- Sobre la evaluación, esta se hace de manera individual y grupal, es decir, aunque nadie aprende por el otro, el aprendizaje es una responsabilidad individual, sin embargo, se consideran los espacios en los que la evaluación se realiza de manera grupal.

\section{Procedimiento metodológico}

La implementación de la innovación educativa que se plasma en este artículo y que permitió mejorar la integración de teoría y práctica en el curso, se enmarca dentro de un enfoque cualitativo y responde a un diseño de investigación-acción. Ya que el objetivo de este trabajo es la atención a una necesidad encontrada en el proceso de aprendizaje de los estudiantes de la carrera, por lo tanto, al sistematizar las debilidades encontradas en la construcción de los conocimientos en relación con la logística, se procedió a indagar en diversas fuentes bibliográficas, como una estrategia o metodología que permitiera mejorar la adquisición de los conceptos por parte del estudiantado.

De acuerdo con Lomax (1990, p.24) citado por Antonio Latorre (2003), la investigaciónacción es una intervención en la práctica profesional con intención de ocasionar alguna mejora. La intervención se basa en la investigación debido a que implica una indagación.

Asimismo, como menciona Kemmis y McTaggart (1988, p.25) señalado por Antonio Latorre (2003), la investigación-acción presenta las siguientes características:

- Es participativa, las personas trabajan para mejorar sus propias prácticas.

- Es colaborativa, se realiza en grupo por las personas implicadas.

- Crea comunidades autocríticas de personas que participan y colaboran en todas las fases del proceso de investigación

- Es un proceso sistemático de aprendizaje, orientado a la praxis (acción críticamente informada y comprometida) 
- Induce a teorizar sobre la práctica

- Somete a prueba las prácticas, las ideas y las suposiciones.

- Implica registrar, recopilar y analizar nuestros propios juicios.

En este sentido, la enseñanza se visualiza como una actividad investigadora por parte del profesor, lo que transciende en la búsqueda constante de mejoras en la práctica pedagógica que este realiza y su vez contribuir en la calidad educativa.

En relación con los instrumentos aplicados para aplicar la innovación educativa, se realizaron quince sesiones grupales para un grupo de treinta estudiantes, las cuales fueron sistematizadas mediante notas crudas para su respectivo análisis. En el siguiente apartado se profundiza los pasos metodológicos implementados.

\section{Contextualización de la Innovación}

Antes de la aplicación de la estrategia, se desarrollaron espacios de sensibilización con los estudiantes a partir de contextualización de la realidad: en este caso fue indispensable identificar un entorno industrial/comercial de fácil acceso para el estudiantado, con el objetivo de que visualizara el sentido práctico y funcional de la teoría (en este caso la industria de restaurantes). En años anteriores el estudiantado del curso, eran quienes buscaban una empresa por sus propios medios, con el propósito de desarrollar el trabajo de campo, sin embargo, no siempre las empresas daban acceso a la población estudiantil sin experiencia limitando así la construcción de conocimiento.

Posteriormente, en complemento con la estrategia de sensibilización anterior, se plantea una estrategia de aprendizaje cooperativo constructivista como innovación educativa. En este sentido, de acuerdo con los principios de la implementación de este tipo de aprendizaje, se pretende que el estudiantado logre una mejor aprehensión de los conceptos y logre su respectiva aplicación en un entorno de trabajo real al tener la tarea de construir un Modelo de Negocio a detalle.

Específicamente, la innovación educativa fue implementada en un grupo de 30 estudiantes, matriculados en el curso Logística I (II-0605), el cual pertenece al tercer año de la Carrera de Ingeniería Industrial. Dicha innovación se realizó de manera paralela a las 15 clases teóricas del curso, en donde a lo largo de las mismas el docente impartiría las diferentes temáticas teóricas que el estudiante debería dominar para ir armando su proyecto.

A continuación se detalla la aplicación de los instrumentos aplicados, es decir los talleres y sesiones grupales para implementar la estrategia didáctica:

\section{Paso 1: Construcción Grupal y cooperación intra-grupal:}

En la primera semana del curso los 30 estudiantes fueron distribuidos en cinco grupos de trabajo. Cada grupo de trabajo tenía por tarea construir su propio Modelo de Negocio, mediante cooperación intra-grupal. De acuerdo con la asignación de roles específicos para cada uno de 
los integrantes y de manera cooperativa, lograron investigar las principales variables para construir un negocio. A partir de esta experiencia se logró una sintonía sobre las realidades concretas de la logística en una realidad concreta. El Modelo de Negocio propuesto fue el de un Restaurante a inaugurar en las inmediaciones de San Pedro o Curridabat. En este caso, se consideraron los restaurantes deportivos, los cuales usualmente proyectan diversos tipos de juegos y competencias deportivas en pantalla gigante con el objetivo de atraer el público. Esta modalidad de negocio comercial fue elegida por las siguientes razones:

i Es un negocio que todos los estudiantes conocen,

ii La aplicación práctica de la teoría en un entorno real, en este sentido, un restaurante que promueve partidos de fútbol, baloncesto, carreras de atletismo, entre otros, presume de gusto por parte de los estudiantes,

iii La recopilación de la información puede derivarse de varias fuentes reales, es decir, no es una fuente única ni restrictiva de información,

iv El estudiante se enfrenta a un reto real y se motiva a investigar de manera independiente y autónoma.

En cada una de las clases impartidas por el profesor se exponía la teoría logística del curso y se debatía en clase como esta teoría aplicaba para el caso del restaurante. La idea del debate en clase surge como elemento alineador de criterios entre los cinco grupos y a la vez como eje aclaratorio de la teoría que se daba semana a semana. Sirvió mucho esta idea como elemento catalizador en el estudiante para que fuese identificando como lo que se veía en cada clase no era teoría aislada, sino que tenía un potencial de aplicación a su caso de negocio. Un rol fundamental era el debate y aporte que cada grupo hacía en la clase, de acuerdo al avance que llevaba de su Modelo de Negocios que iba construyendo, es decir, los mismos estudiantes aportaban y explicaban a sus colegas.

De acuerdo con los contenidos y objetivos del trabajo de campo del curso, se les solicitó a los estudiantes construir un simulador de escenarios como parte de su propuesta de Modelo de Negocio, que incluyera los siguientes componentes:

i Un plan logístico a partir de los elementos básicos:

a. Configuración de la Cadena de Abastecimiento

b. Respuesta al Cliente,

c. Administración de Inventarios,

d. Abastecimiento,

e. Transporte y

f Almacenamiento

ii Gastos operativos e inversión

iii Proyección del estado de pérdidas y ganancias con visión a cinco años (evaluación financiera). 
iv Un simulador/modelo programado para evaluar variables logísticas sensibles y escenarios "que pasa si" (what-if scenarios).

La construcción de cada modelo de negocio se hizo a partir del aprendizaje cooperativo, en el cual el estudiantado indaga detalles y construye el modelo de negocio de acuerdo con las variables logísticas definidas para el esquema de trabajo. Como fuentes de información, además del profesor y los contenidos teóricos de sus lecciones, se fomentan: observación de otros modelos de negocios homólogos, entrevistas con expertos en el tema, potenciales proveedores, estándares de la industria, entre otros.

En este caso, la recolección de datos fue un proceso real y formativo, debido a que se requería que la información estuviese justificada con la debida prueba de documentación. Cabe resaltar que esta etapa de construcción de información, se implementó bajo la modalidad de "cooperación intra-grupal", es decir, cada uno de los cinco grupos debía construir su propuesta mediante cooperación de cada uno de sus integrantes y mediante la planificación de las diversas tareas que se debían realizar para la respectiva construcción.

\section{Paso 2: Presentación Grupal de la propuesta del Modelo de Negocio}

En la semana 10 del curso se pidió la presentación formal del avance del Modelo de Negocio. En esta sesión cada uno de los cinco grupos presentó su avance al profesor y a sus colegas. El objetivo de esta sesión era una alineación conceptual y un debate participativo de los grupos ante lo que propone el grupo expositor. En esta sesión se detectaron muchos detalles a corregir, detalles que no solo el profesor comentaba, sino los mismos compañeros colegas a partir de las propuestas que ellos habían realizado.

\section{Paso 3: Taller Cooperativo entre grupos para calibración de la propuesta del Modelo de Negocio}

En la semana 11 se diseñó un Taller Cooperativo entre los grupos. En este taller lo que se perseguía es que mediante comparación de indicadores claves de cada propuesta de negocio, cada grupo pudiera estudiar si su propuesta hacía sentido racional cuando la comparaba con sus grupos homólogos, esto a partir de la lógica de que los Modelos de Negocios deberían ser homólogos o racionalmente parecidos.

Es decir, a lo largo de las 10 semanas se promovió la cooperación dentro de los grupos y durante el Taller Cooperativo de semana 11 se promovió la cooperación entre los diferentes grupos.

El objetivo de este taller es compartir enfoques innovadores y detalles logísticos que los grupos lograron identificar al compartir y comparar con sus grupos pares, lo cual permitiría que estos generaran conocimiento y se visualizará una mejor apropiación de los conceptos, permitiendo que cada grupo retroalimentara su modelo de propuesta de negocio y así implementara mejoras al modelo durante las siguientes semanas de construcción. 
Esta sesión de Taller Cooperativo se realizaba en torno a la Tabla comparativa del perfil de las propuestas de restaurante (ver tabla 1), mediante el cual el estudiantado a través de comparación de indicadores claves de su propuesta, con la propuestas de sus grupos colegas, podían auto evidenciar donde debía gestionar, afinar y ejecutar correcciones a sus modelos planteados. Las correcciones que cada grupo encontró necesarias hacer las implementó en las restantes 5 semanas de trabajo.

Específicamente cada grupo construyó un cuadro del Perfil del Restaurante según su propuesta, esta construcción la llevó a cabo previamente a la sesión del Taller Cooperativo. $\mathrm{Al}$ iniciar el taller se consolidó un solo cuadro lleno con cada una de las columnas propuestas por los diversos grupos. A partir de la comparación de cada uno de los perfiles de las diversas propuestas se generó la sesión de trabajo. El eje central de dicho taller se fundamentó en la comparación racional de los perfiles de los modelos, para que los estudiantes retroalimentaran y mejoraran los procesos obtenidos. Es decir, debido a que todos los grupos estaban proponiendo un mismo caso de negocio, es de esperar que las propuestas fueran semejantes o al menos homólogas, por lo que si en la sesión de comparación se detectaban diferencias notables, estas daban lugar a estudio, comparación, debate y ajuste de los modelos. En este caso, se utilizaron preguntas abiertas y estructuradas que fomentaban el análisis de las perfiles de las propuestas de negocios. Por lo tanto, mediante la comparación de los cuadros de cada grupo, el estudiantado reflexionaba si su modelo se adecuaba a un contexto real o si requería de algún ajuste; lo interesante de la innovación es que la retroalimentación entre grupos se realizaba de manera cooperativa entre los mismos estudiantes, dejando de lado la necesidad de recurrir al profesor. El profesor era el último recurso mediador. 
Tabla 1:

Tabla comparativa del perfil de las propuestas de restaurante

\section{Perfil de Propuestas de Restaurante}

\begin{tabular}{|c|c|c|c|c|}
\hline Rubro & Grupo 1 & Grupo 2 & Grupo 3 & Grupo 4 \\
\hline \multicolumn{5}{|l|}{ Gastos (anual) } \\
\hline Salarios y Cargas & - & - & - & - \\
\hline Alquiler del local & - & - & - & - \\
\hline Telefono & - & - & - & - \\
\hline Electricidad & - & - & - & - \\
\hline Materiales Empaque & - & - & - & - \\
\hline MRO, Suministros Limpieza & - & - & - & - \\
\hline Mantenimiento de Edificio & - & - & - & - \\
\hline Vigilancia & - & - & - & - \\
\hline Total Gastos Operativos & - & - & - & - \\
\hline \multicolumn{5}{|l|}{ Caracteristicas del Negocio } \\
\hline Cantidad de Personal (fijo) & - & - & - & - \\
\hline Cantidad de Personal (temporal) & - & - & - & - \\
\hline Cantidad de SKUs comercializados & - & - & - & - \\
\hline Cantidad de SKUs "preparados" & - & - & - & - \\
\hline Cantidad de Categorias SKUs comercializados & - & - & - & - \\
\hline Cantidad de Categorías SKUs "preparados" & - & - & - & - \\
\hline Ventas Anuales & - & - & - & - \\
\hline Utilidad Bruta Anual & - & - & - & - \\
\hline Utilidad Neta Anual & - & - & - & - \\
\hline Cantidad de Proveedores & - & - & - & - \\
\hline Frecuencia de visita de Proveedor mas frecuente & - & - & - & - \\
\hline Frecuencia de visita de Proveedor menos frecuente & - & - & - & - \\
\hline Tamaño del Local (m2) & - & - & - & - \\
\hline Cantidad de Parqueos & - & - & - & - \\
\hline Inversión Inicial & - & - & - & - \\
\hline Porcentaje Margen Promedio por SKU & - & - & - & - \\
\hline \multicolumn{5}{|l|}{ Caracteristicas del menu } \\
\hline Bebida más vendida en el Restaurante & - & - & - & - \\
\hline Precio de venta de la Bebida más vendida & - & - & - & - \\
\hline Costo de la Bebida más vendida & - & - & - & - \\
\hline Margen Unitario de la Bebdida más vendida & - & - & - & - \\
\hline Plato más vendido en el Restaurante & - & - & - & - \\
\hline Precio de venta del Plato más vendido & - & - & - & - \\
\hline Costo del Plato más vendido & - & - & - & - \\
\hline Margen Unitario del Plato más vendido & - & - & - & - \\
\hline Cantidad de SKUs de Postres & - & - & - & - \\
\hline Cantidad de SKUs de Entradas & - & - & - & - \\
\hline
\end{tabular}

Nota: Elaboración propia.

\section{Paso 4: Presentación Final del Modelo de Negocio}

Por último, en la semana 18, cada grupo debía realizar una presentación final de 45 minutos para trasmitir a un tercero de manera exitosa la propuesta comercial. En este 
sentido, la propuesta de negocio debía ser presentada a un supuesto inversionista extranjero (este rol lo cumple el profesor y los grupos restantes de la clase) de manera tal que permitiera promocionar el modelo de negocio de manera exitosa y concreta en un tiempo pre-definido. Es en esta sesión donde ya se presenta la propuesta madura del grupo ya que ha pasado por previas fases de calibración, ajuste y revisión. Es acá donde el estudiante se presenta no solo como experto en las variables del caso del restaurante, sino que ya domina los conceptos teóricos logísticos del curso, pues los tuvo que tener claros antes de implementarlos en la solución del caso de armado del restaurante.

Es importante señalar que como parte de la estrategia de sensibilización, la cual se llevó a cabo de forma paralela a la implementación de la estrategia de aprendizaje cooperativo, el profesor relacionó la teoría desarrollada en cada una de las clases en situaciones contextualizadas a la industria de este tipo de restaurantes. Es decir, cada contenido fue objeto de preguntas exploratorias dirigidas al estudiantado durante las lecciones, para visualizar cómo estos podrían implementar los conceptos en el modelo de negocio que paulatinamente construyen a lo largo del curso. Lo ideal en la estrategia es que el profesor no se presente como "experto" en el negocio y que sea el alumnado el que comparta con él las aplicaciones en el esquema de trabajo.

Asimismo, fue importante el desarrollo del criterio experto que fue generado por el estudiantado conforme avanzaba en el conocimiento de la logística de esta industria. Esto se fomentaba sistemáticamente en las clases. Por lo que es importante fomentar que sean los mismos estudiantes de otros grupos los que complementen y juzguen los comentarios de sus compañeros, como parte del aprendizaje cooperativo.

Es importante resaltar que esta estrategia didáctica es efectiva, en la medida en que el alumnado comprenda los conceptos teóricos logísticos y logre integrarlos en la práctica, en este sentido, la realidad empresarial del sector industrial que se estudia.

Además cabe señalar, que los estudiantes tenían libertad al elegir las fuentes de información y la forma de cómo estructurar el negocio. El estudiantado fue autónomo en el proceso de construcción del conocimiento. El profesor controlaba los avances del trabajo mediante sesiones en donde cada grupo exponía a sus pares; incluso los grupos pares aportaban a este control al cuestionar a sus pares. En estos detalles está la complejidad del proyecto.

\section{Resultados de la aplicación de la innovación:}

Considero que como docente en el área de ingeniería la propuesta de trabajo no es común en la carrera, en este sentido, los estudiantes del curso en un principio se mostraban con dudas, pues están acostumbrados a seguir guías de trabajo con actividades más exactas o de pasos más estructurados. Es decir, en este caso se exponen a como es en realidad la vida profesional, en donde se exponen a situaciones en donde hay que diseñar a partir de "una hoja en blanco" en vez de seguir instrucciones y pasos preestablecidos por el profesor.

Dentro de las calificaciones emitidas por el estudiantado respecto a la implementación del Taller Cooperativo y la creación del Modelo de Negocio se encontraron las siguientes: 
- La actividad sirvió para identificar en el proceso errores conceptuales y prácticos en las propuestas de trabajo. Por ejemplo, se encontraron productos que tenían cálculos incorrectos, por lo que se producían pérdidas en vez de ganancias.

- La cooperación entre grupos, la comparación de la información clave y la presión por generar un buen resultado y a tiempo, generó el ambiente propicio para integrar los conceptos en la práctica y sobre todo cooperar.

- La utilización de una plantilla con variables específicas (cuadro comparativo), dotó al estudiante de un listado de los puntos más importantes a verificar a la hora de validar el modelo y someterlo a comparación con los demás grupos.

- Debido a que la programación del modelo se realizó en MS Excel, luego de la sesión cooperativa fue posible realizar ajustes a los cálculos, lo cual resultó en mayor precisión del modelo y mejor entendimiento del mismo.

- Mucha de la discusión a partir de la comparación derivó cuestionamientos sobre las diferentes fuentes de información de los grupos y el tratamiento de la información. Esto permitió profundizar en conocimiento a un mejor nivel.

- La sesión de cooperación entre grupos permitió el análisis de diversas soluciones a las fallas detectadas en los modelos de negocio.

- Un grupo comentó: "Fue muy beneficioso poder compartir con el resto de compañeros sus experiencias con el simulador, en especial la parte en que se intercambió información cuando un grupo estaba débil en un aspecto y otro que estaba fuerte ayudó a brindar un camino de solución basados en sus experiencias".

- Otro grupo comentó: "Nos sentimos expertos en el tema y por primera vez presentamos el trabajo a otros profesionales y hablamos de detalles como expertos, con propiedad".

Asimismo, la actividad permitió esclarecer algunos aspectos secundarios peroimportantes, tales como: el manejo de productos perecederos, los métodos de reaprovisionamiento de las simulaciones, políticas de recambio de uniformes a los empleados, logística de parqueo para los visitantes, la utilización de los materiales de empaque, mejora en los pronósticos del negocio y diseño de frecuencia respecto a la visita de proveedores claves, entre otros.

A nivel general, se visualizó una mejoría en los informes de los estudiantes y la presentación oral del Modelo de Negocio. En este caso, la planificación y la sistematización del proceso de aprendizaje, marcaron la diferencia en relación con otros grupos de estudiantes que han matriculado el mismo curso. La gran diferencia en este aspecto la marca el dominio que genera el grupo sobre la realidad concreta que se estudia y la presión colectiva que genera la cooperación de grupo, pues para colaborar hay que entender el detalle de la materia y como aplicarla.

\section{Conclusiones}

A nivel general, se presentan las siguientes conclusiones:

- El aprendizaje cooperativo funcionó como eje fundamental en la innovación de 
la estrategia didáctica, permitiendo en los estudiantes de Ingeniería Industrial la construcción de conocimiento en torno a los conceptos teóricos de logística al aplicarlos correctamente en una realidad práctica empresarial.

- La realidad práctica empresarial a la cual se enfocó este proyecto, tiene la particularidad que posee ejes homólogos de otras industrias, por lo que el conocimiento generado por los estudiantes es fácilmente replicables a otras industrias. Con esto se garantiza que el alumnado sea capaz de replicar su conocimiento en otras realidades empresariales, y sobre todo que quede capacitado a enfrentar los retos de complejidad logística a través de la cooperación con otras personas que pueden compartirle información clave que le guíe más rápidamente hacia la solución que debe implementar.

- El aprendizaje del estudiantado se mostró más ágil y de mayor impacto a la hora de implementar esta metodología, pues se logra que el estudiante relacione la teoría explicada en clase con la práctica. Al inicio esta puesta en práctica es difícil pero mediante la cooperación entre el alumnado fue aclarándose y mostrándose fácil.

- La aplicación de la teoría en la práctica permite que el alumnado no memorice la materia, sino que entienda los principios de la misma y su aplicación. Al enfrentarse a una problemática real y compleja, recurre automáticamente a la cooperación para sacar la tarea a tiempo en forma y fondo, lo cual le da competencias valiosas para futuros trabajos en equipo.

- El trabajo cooperativo fomenta la construcción del conocimiento y permite a la persona modificar lo que ya sabe con la adquisición de otros conceptos a través de la cooperación y comparación. En este caso quedó muy evidente que el profesor no es el centro del conocimiento, sino más bien es la interacción cooperativa entre los participantes del grupo la que funda la generación de conocimiento.

- Socializar el conocimiento permitió que los mismos estudiantes permearan a sus colegas de conocimientos que habían adquirido, siendo este un método incluso más efectivo que una clase magistral o métodos convencionales. A partir de este método de trabajo se hizo más fácil la explicación de la teoría, pues la teoría no fue vista como una simple materia de clase, sino como algo necesario para gestionar el negocio que se estaba diseñando. En este aspecto la cooperación entre los compañeros incluso facilitó la asimilación de los conceptos.

- La experiencia en cuestión puede ser replicada horizontalmente hacia otros cursos de la carrera de Ingeniería Industrial, incluso en otras carreras, sirviendo la metodología medular a otras facultades.

\section{Referencias}

Amaya, L.E. (s.f.). Aprendizaje colaborativo orientado a proyectos en el curso álgebra lineal para informática empresarial. Recuperado de www.cibem.org/.../306_1372310550_extenso_del_ documento.docx 
Baumberger-Henry, M. (2005). Cooperative learning and case study: does the combination improve student's perception of problem-solving and decision making skills? [Aprendizaje cooperativo y caso de estudio: ¿̇la combinación de ambos mejora las habilidades del estudiante respecto a solución de problemas y toma de decisiones?] Nurse Education Today, 25, 238-246.

Ferreiro, R., Calderón, M. (2007). El ABC del aprendizaje cooperativo. México: Editorial Trillas.

Li, L.K.Y. (2001). Some refinements on peer assessment of group projects. [Algunos ajustes sobre la evaluación de pares a proyectos en grupo.] Assessment and Evaluation in Higher Education 26 (1), 5-8 Recuperado de http://www.tandfonline.com/doi/abs/10.1080/02602 93002002255\#preview

Smagorinsky, P. (2007). Vigotsky and the Social Dynamics of Classrooms [Vigotsky y la Dinámica Social de la Clase]. English Journal, 97(2), 61-66.

Tenutto, M. y Klinoff, A. (2005). Escuela para Maestros. Argentina: Editorial Circulo Latino Austral.

Latorre, Antonio. (2003). La investigación-acción. Conocer y cambiar la practica educativa. Madrid: Editorial GRAO.

Ferreiro, R., Calderón, M. (2007). El ABC del aprendizaje cooperativo. México: Editorial Trillas. 